\title{
Detection and Imaging of Multiple Ground Moving Targets using Ultra-Narrowband Continuous-Wave SAR
}

\author{
Ling Wang $^{a}$ and Birsen Yazıc1 ${ }^{b}$ \\ ${ }^{a}$ Department of Information and Communication Engineering, Nanjing University of \\ Aeronautics and Astronautics, Nanjing, Jiangsu 210016 China; \\ ${ }^{b}$ Department of Electrical, Computer and System Engineering, Rensselaer Polytechnic \\ Institute, Troy, NY 12180 USA
}

\begin{abstract}
We present a novel method for ground moving target detection and imaging using a SAR system transmitting ultra-narrowband continuous waveforms. We develop a new forward model that relates the velocity as well as reflectivity information at each location to a correlated received signal. We reconstruct moving target images by a filtered-backprojection method. We use the image contrast as a metric to detect moving targets and to determine their velocities. The method results in well-focused reflectivity images of moving targets and their velocity estimates regardless of the target location, speed, and velocity direction. We present numerical experiments to verify our method.
\end{abstract}

Keywords: Moving Target, Detection, Imaging, Synthetic Aperture, Continuous Wave (CW), Doppler

\section{INTRODUCTION}

Ground moving target detection and imaging using synthetic aperture radar (SAR) has received considerable attention in the past two decades. ${ }^{1-17}$ However, all the existing techniques are for traditional synthetic aperture radar (SAR) systems, which accomplish high-range resolution imaging of static scenes and moving targets by transmitting wideband waveforms. We consider the problem of synthetic aperture moving target detection and imaging using ultra-narrowband CW waveforms. Such a SAR system requires relatively simple and lowcost transmitter, and in some cases does not need a dedicated transmitter. Existing radio frequency signals, such as radio, television signals, WiFi signals, etc. can be used as the transmission sources. Additionally, ultra-narrowband continuous waveforms have high Doppler resolution and are capable of capturing the velocity information of the moving targets as compared to the high range resolution waveforms used by the traditional SAR systems.

In [18], we presented a novel synthetic aperture imaging method of stationary scenes that takes advantage of the high Doppler resolution of the transmitted ultra-narrowband continuous waveforms. In this paper, we consider the moving target detection and imaging problem using such SAR systems. Our approach exploits the high Doppler resolution of the transmitted waveforms to reconstruct the reflectivity (position), as well as to estimate the velocity field of moving targets. We develop a novel model that relates the velocity field and the reflectivity of the scene to a correlated received signal and a corresponding FBP-type novel image formation method. A set of FBP images are reconstructed with hypothesized velocities that cover the expected velocity range. Moving targets are well focused in the reflectivity images corresponding to their velocities. We use the image contrast as a metric to detect targets and to determine their velocities. We present numerical experiments to demonstrate the performance of the moving target detection and imaging method. To the best of our knowledge, our method is the first in the literature that addresses the ground moving target detection and imaging using such SAR systems.

The organization of the paper is as follows. In Section 2, we present the model for the received signal and develop the forward model for moving target imaging using ultranarrow-band CW waveforms. In Section 3,

Further author information: (Send correspondence to B.Y.)

B.Y.: E-mail: yazici@ecse.rpi.edu, Telephone: +1(518)276 2905, Fax: +1(518)276 6261

L.W.:E-mail: wanglrpi@gmail.com

Algorithms for Synthetic Aperture Radar Imagery XIX, edited by Edmund G. Zelnio, Frederick D. Garber, Proc. of SPIE Vol. 8394, 83940H · C 2012 SPIE · CCC code: 0277-786X/12/\$18 · doi: 10.1117/12.918991 
we develop an FBP-type image formation method to reconstruct the reflectivity of the scene and a contrastmaximization based velocity estimation method. In Section 4, we present numerical simulations to demonstrate the performance of the proposed moving target imaging method. Section 5 concludes our paper.

We use the following notational conventions throughout the paper. The bold Roman, bold italic and Roman lower-case letters are used to denote variables in $\mathbb{R}^{3}, \mathbb{R}^{2}$ and $\mathbb{R}$, respectively, i.e., $\mathbf{z}=(\boldsymbol{z}, z) \in \mathbb{R}^{3}$, with $\boldsymbol{z} \in \mathbb{R}^{2}$ and $z \in \mathbb{R}$. The calligraphic letters $(\mathcal{F}, \mathcal{K}$ etc. $)$ are used to denote operators.

\section{FORWARD MODEL FOR MOVING TARGET IMAGING}

\subsection{Received Signal}

Let $\mathbf{x}=(\boldsymbol{x}, \psi(\boldsymbol{x})) \in \mathbb{R}^{3}$ denote the earth's surface, where $\boldsymbol{x} \in \mathbb{R}^{2}$ and $\psi: \mathbb{R}^{2} \rightarrow \mathbb{R}$ is a known function for the ground topography. Let $\mathbf{v}_{\mathbf{x}}$ denote the velocity of the moving scatterer located at $\boldsymbol{x}$ at $t=0$. Then,

$$
\mathbf{v}_{\mathbf{x}}=\left[\boldsymbol{v}_{\mathbf{x}}, \nabla_{\boldsymbol{x}} \psi(\boldsymbol{x}) \cdot \boldsymbol{v}_{\mathbf{x}}\right]
$$

where $\boldsymbol{v}_{\mathbf{x}} \in \mathbb{R}^{2}$ is the two dimensional velocity and $\nabla_{\boldsymbol{x}} \psi(\boldsymbol{x})=\left[\frac{\partial \psi}{\partial x_{1}} \quad \frac{\partial \psi}{\partial x_{2}}\right]$. Note that $\mathbf{v}_{\mathbf{x}}$ may be $t$ dependent. In the rest of our discussion, we make the assumption that the scatterers are moving linearly and therefore the velocity $\mathbf{v}_{\mathbf{x}}$ is time independent. Furthermore, we assume that the functional form of $\mathbf{v}_{\mathbf{x}}$ does not depend on $\mathbf{x}$. It is simply a constant velocity for each $\mathbf{x}$. Thus, at time $t$, the scatter is located at $\mathbf{z}=\mathbf{x}+\mathbf{v}_{\mathbf{x}} t$.

We assume that the scattering takes place in a thin region near the ground. Thus, the reflectivity function of the scene at time $t=0$ has the form

$$
V(\mathbf{x})=\rho(\boldsymbol{x}) \delta(\boldsymbol{x}-\psi(\boldsymbol{x})) .
$$

At time $t$, taking into account the movement of the scatters, the reflectivity function of the moving scatterers translates as

$$
V\left(\mathbf{z}-\mathbf{v}_{\mathbf{x}} t\right) \simeq \rho\left(\boldsymbol{z}-\boldsymbol{v}_{\mathbf{x}} t\right) \delta\left(z_{3}-\psi(\boldsymbol{z})\right) \delta\left(v_{3}-\nabla_{\boldsymbol{z}} \psi(\boldsymbol{z}) \cdot \boldsymbol{v}_{\mathbf{x}}\right) .
$$

Let $\gamma_{T}(t)$ and $\gamma_{R}(t)$ be the transmitter and receiver trajectories, respectively; and $p(t)$ denotes the transmitted waveform and $r(t)$ denote the received signal. We consider a finite and relatively short receiving time-window starting at time $t=s$.

Using the scalar wave equation along with (3) and under the Born approximation, taking into account the fact that the velocities of the antennas and targets are much less than the speed of light; and under the assumption that the range variation due to the movement of the antennas and the scene is much less than the the transmitter-to-scene and scene-to-receiver range, we have ${ }^{18}$

$$
r(t+s)=-\omega_{0}^{2} \int \frac{\tilde{p}(\alpha t-\tau+s) \mathrm{e}^{\mathrm{i} \omega_{0}(\alpha t-\tau+s)} q(\boldsymbol{x}, \boldsymbol{v})}{(4 \pi)^{2} G_{T R}(s, \boldsymbol{x}, \boldsymbol{v})} d \boldsymbol{x} d \boldsymbol{v}
$$

where $q(\boldsymbol{x}, \boldsymbol{v})=\rho(\boldsymbol{x}) \delta\left(\boldsymbol{v}-\boldsymbol{v}_{\mathbf{x}}\right)$ is the phase-space reflectivity function of the moving scene. Note that a narrowband waveform is assumed in (4), i.e., $p(t)=\mathrm{e}^{\mathrm{i} \omega_{0} t} \tilde{p}(t)$ where $\omega_{0}$ denotes the carrier frequency and $\tilde{p}(t)$ is the complex envelope of $p$, which is slow varying as a function of $t$ as compared to $\mathrm{e}^{\mathrm{i} \omega_{0} t}$.

The time dilation $\alpha$ in (4) is given by

$$
\alpha=\frac{\left.1-\gamma_{R}(s) \widehat{-(\mathbf{x}}+\mathbf{v} s\right) \cdot \dot{\gamma}_{R}(s) / c_{0}}{\left.1+\gamma_{T}(s) \widehat{-(\mathbf{x}}+\mathbf{v} s\right) \cdot \dot{\gamma}_{T}(s) / c_{0}} \cdot \frac{\left.1+\gamma_{T}(s) \widehat{-(\mathbf{x}}+\mathbf{v} s\right) \cdot \mathbf{v} / c_{0}}{\left.1-\gamma_{R}(s) \widehat{-(\mathbf{x}}+\mathbf{v} s\right) \cdot \mathbf{v} / c_{0}},
$$

the time delay $\tau$ is given by

$$
\left.\left.\tau \approx\left[\left|\gamma_{T}(s)-(\mathbf{x}+\mathbf{v} s)\right|+\left|\gamma_{R}(s)-(\mathbf{x}+\mathbf{v} s)\right|\right] / c_{0}-\left[\left(\gamma_{T}(s) \widehat{-(\mathbf{x}}+\mathbf{v} s\right)+\gamma_{R}(s) \widehat{-(\mathbf{x}}+\mathbf{v} s\right)\right) \cdot \mathbf{v} s\right] / c_{0}
$$


and $G_{T R}$ is the product of the geometrical spreading factors given by

$$
G_{T R}(s, \boldsymbol{x}, \boldsymbol{v})=\left|\gamma_{R}(s)-(\mathbf{x}+\mathbf{v} s)\right|\left|\mathbf{x}+\mathbf{v} s-\gamma_{T}(s)\right| .
$$

Since $\tilde{p}$ is a slow-varying function of time, we approximate $\tilde{p}(\alpha t) \approx \tilde{p}(t)$ in the rest of our discussion.

Note that the time dilation factor $\alpha$ can be approximated as $\alpha=1+\beta$ where $f_{0} \beta\left(f_{0}=\omega_{0} / 2 \pi\right)$ represents the total Doppler frequency induced by the relative radial motion of the antennas and the moving scatters. We refer to it as the bistatic Doppler frequency for moving targets and denote it with $f_{d}(s, \boldsymbol{x}, \boldsymbol{v})$, i.e.,

$$
\left.\left.f_{d}(s, \boldsymbol{x}, \boldsymbol{v})=\frac{f_{0}}{c_{0}}\left[\gamma_{T}(s) \widehat{-(\mathbf{x}}+\mathbf{v} s\right) \cdot\left(\dot{\gamma}_{T}(s)-\mathbf{v}\right)+\gamma_{R}(s) \widehat{-(\mathbf{x}}+\mathbf{v} s\right) \cdot\left(\dot{\gamma}_{R}(s)-\mathbf{v}\right)\right] .
$$

Note that in (7) and (8), $\mathbf{x}=[\boldsymbol{x}, \boldsymbol{\psi}(\boldsymbol{x})]$ and $\mathbf{v}=\left[\boldsymbol{v}, \nabla_{\boldsymbol{x}} \boldsymbol{\psi}(\boldsymbol{x}) \cdot \boldsymbol{v}\right]$.

\subsection{Forward Model}

We define the correlation of the received signal given in (4) with a scaled or frequency-shifted version of the transmitted signal over a finite time window as follows:

$$
d(s, \mu)=\int r(t+s) p^{*}(\mu t) \phi(t) d t
$$

for some $s \in \mathbb{R}$ and $\mu \in \mathbb{R}^{+}$, where $\phi(t), t \in\left[0, T_{\phi}\right]$ is a smooth windowing function with a finite support.

Substituting (4) into (9), we obtain

$$
d(s, \mu)=\int \frac{\mathrm{e}^{\mathrm{i} \omega_{0}(\alpha-\mu) t} \mathrm{e}^{\mathrm{i} \omega_{0}(s-\tau)}}{(4 \pi)^{2} G_{T R}(s, \boldsymbol{x}, \boldsymbol{v})} \omega_{0}^{4} \tilde{p}(t-\tau+s) \tilde{p}^{*}(t) q(\boldsymbol{x}, \boldsymbol{v}) d \boldsymbol{x} d \boldsymbol{v} d t .
$$

Note that $\tilde{p}(\mu t) \approx \tilde{p}(t)$ is used in (10).

We define the forward modeling operator, $\mathcal{F}$, as follows:

$$
\begin{aligned}
d(s, \mu) & \approx \mathcal{F}[q](s, \mu) \\
& :=\int \mathrm{e}^{-\mathrm{i} \phi(t, \boldsymbol{x}, \boldsymbol{v}, s, \mu)} A(t, \boldsymbol{x}, \boldsymbol{v}, s, \mu) q(\boldsymbol{x}, \boldsymbol{v}) d \boldsymbol{x} d \boldsymbol{v} d t
\end{aligned}
$$

where

$$
\begin{aligned}
& \phi(t, \boldsymbol{x}, \boldsymbol{v}, s, \mu)=2 \pi f_{0} t\left[(\mu-1)+f_{d}(s, \boldsymbol{x}, \boldsymbol{v}) / f_{0}\right] \\
& A(t, \boldsymbol{x}, \boldsymbol{v}, s, \mu)=\frac{\tilde{p}(t-\tau+s) \tilde{p}^{*}(t) \mathrm{e}^{\mathrm{i} \omega_{0}(s-\tau)} \omega_{0}^{4}}{(4 \pi)^{2} G_{T R}(s, \boldsymbol{x}, \boldsymbol{v})}
\end{aligned}
$$

We assume that for some $m_{A}, A$ satisfies the inequality

$$
\sup _{(t, \mu, s, \boldsymbol{x}, \boldsymbol{v}) \in \mathcal{U}}\left|\partial_{t}^{\alpha_{t}} \partial_{\mu}^{\alpha_{\mu}} \partial_{s}^{\beta_{s}} \partial_{x_{1}}^{\epsilon_{1}} \partial_{x_{2}}^{\epsilon_{2}} \partial_{v_{1}}^{\varepsilon_{1}} \partial_{v_{2}}^{\varepsilon_{2}} A(t, \boldsymbol{x}, \boldsymbol{v}, s, \mu)\right| \leq C_{A}\left(1+t^{2}\right)^{\left(m_{A}-\left|\alpha_{t}\right|\right) / 2}
$$

where $\mathcal{U}$ is any compact subset of $\mathbb{R} \times \mathbb{R}^{+} \times \mathbb{R} \times \mathbb{R}^{2} \times \mathbb{R}^{2}$, and the constant $C_{A}$ depends on $\mathcal{U}, \alpha_{t, \mu}, \beta_{s}, \epsilon_{1,2}, \varepsilon_{1,2}$. This assumption is needed in order to make various stationary phase calculations hold.

\section{IMAGE FORMATION}

We assume that the velocity is constant, say $\boldsymbol{v}_{h}$, and reconstruct a set of two-dimensional reflectivity images in position space for a range of hypothesized velocities $\boldsymbol{v}_{h} \in \Lambda_{v}$. We refer to this image as the $\boldsymbol{v}_{h}$-reflectivity image and form it by an FBP-type imaging operator. We design the filter to ensure that the reconstructed reflectivity for the scatterer at $\boldsymbol{x}$ has the correct strength whenever the hypothesized velocity is equal to the true target velocity, i.e., $\boldsymbol{v}_{h}=\boldsymbol{v}_{\mathbf{x}}$. From this set of images, we estimate the velocity of the targets using a figure of merit that measures the degree to which the images are focused. The reflectivity images corresponding to the estimated velocities provide focused images of the moving targets present in the scene. 


\subsection{FBP- operator}

We form the $\boldsymbol{v}_{h}$-reflectivity image $q_{\boldsymbol{v}_{h}}(\boldsymbol{z})$ for a fixed hypothesized velocity $\mathbf{v}_{h}=\left[\boldsymbol{v}_{h}, \nabla_{\boldsymbol{z}} \boldsymbol{\psi}(\boldsymbol{z}) \cdot \boldsymbol{v}_{h}\right]$ by filtering and backprojecting the data onto the position-space iso-Doppler contour $F_{\boldsymbol{v}_{h}}(s, \mu)$ :

$$
\begin{aligned}
q_{\boldsymbol{v}_{h}}(\boldsymbol{z}) & :=\mathcal{K}_{\boldsymbol{v}_{h}}[d](\boldsymbol{z}) \\
& =\int \mathrm{e}^{\mathrm{i} \phi\left(t, \boldsymbol{z}, \boldsymbol{v}_{h}, s, \mu\right)} Q_{\boldsymbol{v}_{h}}(\boldsymbol{z}, t, s) d(s, \mu) d t d s d \mu,
\end{aligned}
$$

where $\mathcal{K}_{\boldsymbol{v}_{h}}$ is the filtered-backprojection operator for the fixed velocity $\boldsymbol{v}_{h}$ and $Q_{\boldsymbol{v}_{h}}$ is the filter to be determined below. Note that $\boldsymbol{v}_{h}$ is a fixed parameter for $Q_{\boldsymbol{v}_{h}}$.

We assume that for some $m_{Q_{v_{h}}}, Q_{\boldsymbol{v}_{h}}$ satisfies the inequality

$$
\sup _{\left(t, \mu, s, \boldsymbol{z}, \boldsymbol{v}_{h}\right) \in \mathcal{U}}\left|\partial_{t}^{\alpha_{t}} \partial_{\mu}^{\alpha_{\mu}} \partial_{s}^{\beta_{s}} \partial_{z_{1}}^{\epsilon_{1}} \partial_{z_{2}}^{\epsilon_{2}} Q_{\boldsymbol{v}_{h}}\left(t, \boldsymbol{z}, \boldsymbol{v}_{h}, s, \mu\right)\right| \leq C_{Q_{\boldsymbol{v}_{h}}}\left(1+t^{2}\right)^{\left(m_{Q \boldsymbol{v}_{h}}-\left|\alpha_{t}\right|\right) / 2}
$$

where $\mathcal{U}$ is any compact subset of $\mathbb{R} \times \mathbb{R}^{+} \times \mathbb{R} \times \mathbb{R}^{2}$, and the constant $C_{Q_{v_{h}}}$ depends on $\mathcal{U}, \alpha_{t, \mu}, \beta_{s}, \epsilon_{1,2}$. Under the assumption (16), (15) defines $\mathcal{K}_{\boldsymbol{v}_{h}}$ as a Fourier integral operator.

The kernel of the image fidelity operator $\mathcal{K}_{\boldsymbol{v}_{\boldsymbol{h}}} \mathcal{F}$ is the Point Spread Function (PSF) of the two-dimensional reflectivity imaging operator for the hypothesized velocity $\boldsymbol{v}_{h}$ with respect to the true velocity $\boldsymbol{v}_{\mathbf{x}}$, which is given by

$$
L_{\boldsymbol{v}_{h}}^{\boldsymbol{v}_{\boldsymbol{x}}}(\boldsymbol{z}, \boldsymbol{x})=\int \mathrm{e}^{\mathrm{i}\left[\phi\left(t, \boldsymbol{z}, \boldsymbol{v}_{h}, s, \mu\right)-\phi\left(t, \boldsymbol{x}, \boldsymbol{v}_{\mathbf{x}}, s, \mu\right)\right]} Q_{\boldsymbol{v}_{h}}(\boldsymbol{z}, t, s) A\left(t^{\prime}, \boldsymbol{x}, \boldsymbol{v}_{\mathbf{x}}, s, \mu\right) d t d s d \mu d t^{\prime} .
$$

Applying the stationary phase theorem to approximate the $t^{\prime}$ and $\mu$ integrations in $(17){ }^{*}$ and substituting the results back into (17), we get the kernel of the image fidelity operator $\mathcal{K}_{\boldsymbol{v}_{h}} \mathcal{F}$ :

$$
L_{\boldsymbol{v}_{h}}^{\boldsymbol{v}_{\mathbf{x}}}(\boldsymbol{z}, \boldsymbol{x}) \approx \int \mathrm{e}^{\mathrm{i} 2 \pi t\left[f_{d}\left(s, \boldsymbol{z}, \boldsymbol{v}_{h}\right)-f_{d}\left(s, \boldsymbol{x}, \boldsymbol{v}_{\mathbf{x}}\right)\right]} Q_{\boldsymbol{v}_{h}}(\boldsymbol{z}, t, s) A\left(t, \boldsymbol{x}, \boldsymbol{v}_{\mathbf{x}}, s, 1-f_{d}\left(s, \boldsymbol{x}, \boldsymbol{v}_{\mathbf{x}}\right) / f_{0}\right) d t d s .
$$

To simplify our notation, we let

$$
A\left(t, \boldsymbol{x}, \boldsymbol{v}_{\mathbf{x}}, s\right)=A\left(t, \boldsymbol{x}, \boldsymbol{v}_{\mathbf{x}}, s, 1-f_{d}\left(s, \boldsymbol{x}, \boldsymbol{v}_{\mathbf{x}}\right) / f_{0}\right) .
$$

We determine $Q_{\boldsymbol{v}_{h}}$ so that the PSF of the two-dimensional reflectivity imaging operator, $L_{\boldsymbol{v}_{h}}^{\boldsymbol{v}_{\mathbf{x}}}(\boldsymbol{z}, \boldsymbol{x})$ is as close as possible to the Dirac-delta function, $\delta(\boldsymbol{z}-\boldsymbol{x})$ for $\boldsymbol{v}_{h}=\boldsymbol{v}_{\mathbf{x}}$, i.e., whenever the reflectivity at $\boldsymbol{z}$ is reconstructed for the correct target velocity $\boldsymbol{v}_{\mathbf{x}}$. We assume that at the correct target velocity, the flight trajectory and the illumination pattern are chosen such that the only contribution to $L_{\boldsymbol{v}_{h}}^{\boldsymbol{v}_{\mathbf{x}}}(\boldsymbol{z}, \boldsymbol{x})$ comes from those points $\boldsymbol{z}=\boldsymbol{x}$.

Thus, we linearize $f_{d}\left(s, \boldsymbol{z}, \boldsymbol{v}_{h}\right)$ around $\boldsymbol{z}=\boldsymbol{x}$ for $\boldsymbol{v}_{h}=\boldsymbol{v}_{\mathbf{x}}$ and approximate

$$
f_{d}\left(s, \boldsymbol{z}, \boldsymbol{v}_{h}\right)-f_{d}\left(s, \boldsymbol{x}, \boldsymbol{v}_{h}\right) \approx(\boldsymbol{z}-\boldsymbol{x}) \cdot \nabla_{\boldsymbol{z}} f_{d}\left(s, \boldsymbol{z}, \boldsymbol{v}_{h}\right) .
$$

Thus, (18) becomes

$$
L_{\boldsymbol{v}_{h}}^{\boldsymbol{v}_{h}}(\boldsymbol{z}, \boldsymbol{x})=\int \mathrm{e}^{\mathrm{i} t(\boldsymbol{z}-\boldsymbol{x}) \cdot \boldsymbol{\Xi}_{v_{h}}(s, \boldsymbol{z})} Q_{\boldsymbol{v}_{h}}(\boldsymbol{z}, t, s) A\left(t, \boldsymbol{z}, \boldsymbol{v}_{h}, s\right) d t d s
$$

where

$$
\boldsymbol{\Xi}_{\boldsymbol{v}_{h}}(s, \boldsymbol{z})=2 \pi \nabla_{\boldsymbol{z}} f_{d}\left(s, \boldsymbol{z}, \boldsymbol{v}_{h}\right) .
$$

For each $\boldsymbol{z}$, we make the following change of variables:

$$
(t, s) \rightarrow \boldsymbol{\xi}=t \boldsymbol{\Xi}_{\boldsymbol{v}_{h}}(s, \boldsymbol{z})
$$

${ }^{*}$ The determinant of the Hessian of $\Phi_{k}$ is $(2 \pi)^{2} f_{0}^{2}$. Thus, the stationary points are non-degenerate. 
and write (21) as follows:

$$
L_{\boldsymbol{v}_{h}}^{\boldsymbol{v}_{h}}(\boldsymbol{z}, \boldsymbol{x})=\int_{\Omega_{\boldsymbol{v}_{h}, \boldsymbol{z}}} \mathrm{e}^{\mathrm{i}(\boldsymbol{z}-\boldsymbol{x}) \cdot \boldsymbol{\xi}} Q_{\boldsymbol{v}_{h}}(\boldsymbol{z}, \boldsymbol{\xi}) A\left(\boldsymbol{z}, \boldsymbol{v}_{h}, \boldsymbol{\xi}\right) \eta\left(\boldsymbol{z}, \boldsymbol{v}_{h}, \boldsymbol{\xi}\right) d \boldsymbol{\xi}
$$

where

$$
\begin{aligned}
Q_{\boldsymbol{v}_{h}}(\boldsymbol{z}, \boldsymbol{\xi}) & =Q_{\boldsymbol{v}_{h}}(\boldsymbol{z}, t(\boldsymbol{\xi}), s(\boldsymbol{\xi})), \\
A\left(\boldsymbol{z}, \boldsymbol{v}_{h}, \boldsymbol{\xi}\right) & =A\left(t(\boldsymbol{\xi}), \boldsymbol{z}, \boldsymbol{v}_{h}, s(\boldsymbol{\xi})\right)
\end{aligned}
$$

and

$$
\eta\left(\boldsymbol{z}, \boldsymbol{v}_{h}, \boldsymbol{\xi}\right)=\frac{\partial(t, s)}{\partial \boldsymbol{\xi}}=|t|^{-1}\left|\operatorname{det}\left[\begin{array}{c}
\boldsymbol{\Xi}_{\boldsymbol{v}_{h}}(s, \boldsymbol{z}) \\
\partial_{s} \boldsymbol{\Xi}_{\boldsymbol{v}_{h}}(s, \boldsymbol{z})
\end{array}\right]\right|^{-1}
$$

is the determinant of the Jacobian that comes from the change of variables given in (23).

The domain of integration in (24) is given by

$$
\Omega_{\boldsymbol{v}_{h}, \boldsymbol{z}}=\left\{\boldsymbol{\xi}=t \boldsymbol{\Xi}_{\boldsymbol{v}_{h}}(s, \boldsymbol{z}) \mid A\left(t, \boldsymbol{z}, \boldsymbol{v}_{h}, s\right) \neq 0, \quad t, s \in \mathbb{R}\right\} .
$$

We refer to $\Omega_{\boldsymbol{v}_{h}, \boldsymbol{z}}$ as the data collection manifold at $\boldsymbol{z}$ for $\boldsymbol{v}_{h}=\boldsymbol{v}_{\mathbf{x}}$. This set determines many of the properties of the reconstructed reflectivity image when $\boldsymbol{v}_{h}=\boldsymbol{v}_{\mathbf{x}}$.

To approximate the point spread function $L_{\boldsymbol{v}_{h}}^{\boldsymbol{v}_{h}}(\boldsymbol{z}, \boldsymbol{x})$ in $(24)$ with the Dirac-delta function, we choose the filter as follows:

$$
Q_{\boldsymbol{v}_{h}}(\boldsymbol{z}, \boldsymbol{\xi})=\frac{\chi_{\Omega_{\boldsymbol{v}_{h}, \boldsymbol{z}}}}{\eta\left(\boldsymbol{z}, \boldsymbol{v}_{h}, \boldsymbol{\xi}\right)} \frac{A^{*}\left(\boldsymbol{z}, \boldsymbol{v}_{h}, \boldsymbol{\xi}\right)}{\left|A\left(\boldsymbol{z}, \boldsymbol{v}_{h}, \boldsymbol{\xi}\right)\right|^{2}}
$$

where $\chi_{\Omega_{v_{h}, z}}$ is a smooth cut-off function that prevents division by zero in (29).

\subsection{Determination of the moving target velocity}

The filtered-backprojection (FBP) of $d(s, \mu)$ results in a set of reflectivity images $\tilde{q}_{\boldsymbol{v}_{h}}$ in the two-dimensional $\boldsymbol{z}$ space for each velocity value $\boldsymbol{v}_{\boldsymbol{h}}$ for a range of velocities that is suitably chosen for ground moving targets. When the hypothesized velocity $v_{\boldsymbol{h}}$ is equal to the correct velocity $v_{\boldsymbol{z}}$, the corresponding reflectivity image is expected to be well-focused. We measure the degree to which the reflectivity images are focused with the image contrast and generate a contrast-image defined as follows:

$$
I\left(v_{h 1}, v_{h 2}\right)=\mathcal{C}\left[q_{\boldsymbol{v}_{h}}\right]
$$

where $\mathcal{C}$ denotes the contrast operator ${ }^{3}$ and $\boldsymbol{v}_{h}=\left(v_{h 1}, v_{h 2}\right)$ is used as the index of the contrast image. We determine the velocity of the moving scatterers by localizing the local maxima in the contrast-image $I\left(\boldsymbol{v}_{h}\right)$.

\section{NUMERICAL SIMULATIONS}

We considered a scene of size $[0,11] \times[0,11] \mathrm{km}^{2}$ with flat topography centered at $[11,11,0] \mathrm{km}$. The scene was discretized into $128 \times 128$ pixels, where $[0,0,0] \mathrm{m}$ and $[11,11,0] \mathrm{km}$ correspond to the pixels $(1,1)$ and $(128,128)$, respectively. Fig. 1 shows the scene with a static extended target and multiple moving targets along with their corresponding velocities.

We assumed that the transmitter and receiver were traversing a circular trajectory given by $\gamma_{C}(s)=(11+$ $11 \cos (s), 11+11 \sin (s), 6.5) \mathrm{km}$. Let $\gamma_{T}(s)$ and $\gamma_{R}(s)$ denote the trajectories of the two receivers. We set $\gamma_{T}(s)=\gamma_{C}(s)$ and $\gamma_{R}(s)=\gamma_{1}\left(s-\frac{\pi}{4}\right)$. Note that the variable $s$ in $\gamma_{C}$ is equal to $\frac{V}{R} t$ where $V$ is the speed of the receiver, and $R$ is the radius of the circular trajectory. We set the speed of the two receivers to $261 \mathrm{~m} / \mathrm{s}$.

We assumed that the transmitter transmitted a single-frequency continuous waveform operating at $f_{0}=$ $\omega_{0} / 2 \pi=800 \mathrm{MHz}$. We used (4) and (9) to generate the data. The length of the signal was set to $0.1707 \mathrm{~s}$. The circular trajectory was uniformly sampled into 2048 points. 


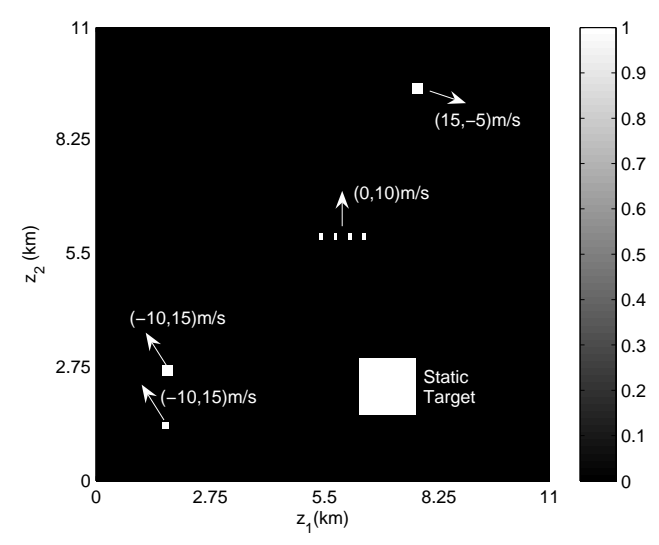

Figure 1: Scene considered in the numerical simulations.

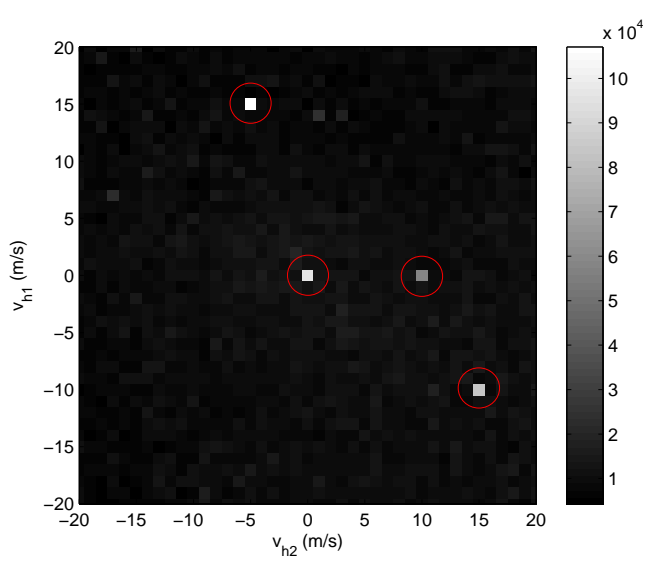

Figure 2: The contrast-image formed by the contrast of the images reconstructed with each hypothesized velocity.

We assumed that the velocity of the targets is in the range of $[-20,20] \times[-20,20] \mathrm{m} / \mathrm{s}$ and reconstructed $q_{\boldsymbol{v}_{h}}(\boldsymbol{z})$ images via the FBP and image-contrast optimization method as described in Section 3. We see from Fig. 2 that there are four dominant peaks marked with red circles in the contrast-image. This indicates that there are four different velocities associated with the targets in the scene. The velocities where the peaks are located are equal to the velocities of the targets we assumed in the simulations.

Fig. 3 presents the reconstructed images using the four estimated velocities. We observe that the targets are well-focused in the image formed using the correct velocity associated with each target.Note that Fig. 3(a) is the image reconstructed with $\mathbf{v}_{h}=[0,0,0] \mathrm{m} / \mathrm{s}$. In this case, the moving target imaging method described here is equivalent to the static target imaging method in. ${ }^{19}$ As expected only the static target is well-focused in Fig. 3(a).

\section{CONCLUSION}

In this paper, we considered the problem of synthetic aperture radar imaging of moving targets using ultranarrowband transmitted waveforms. We presented a received signal model for a dynamic scene. We developed a novel forward model for image formation. We developed an associated novel FBP and image-contrast optimization based image formation method to estimate the velocities of multiple targets and their corresponding reflectivity images. We presented numerical simulations to verify the theoretical results. A complete analysis of the performance of our imaging method and demonstration of its performance in more realistic scenarios will be the focus of our future work. 


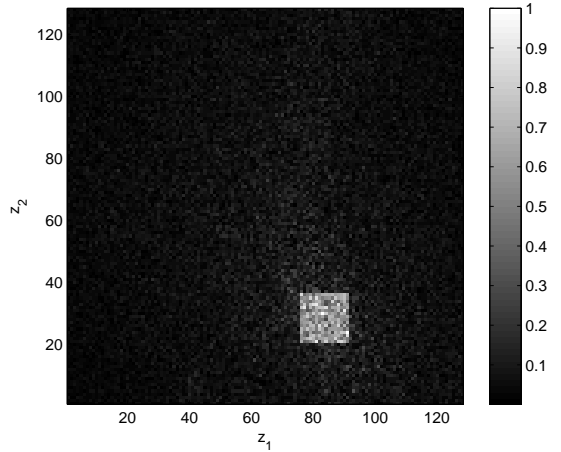

(a)

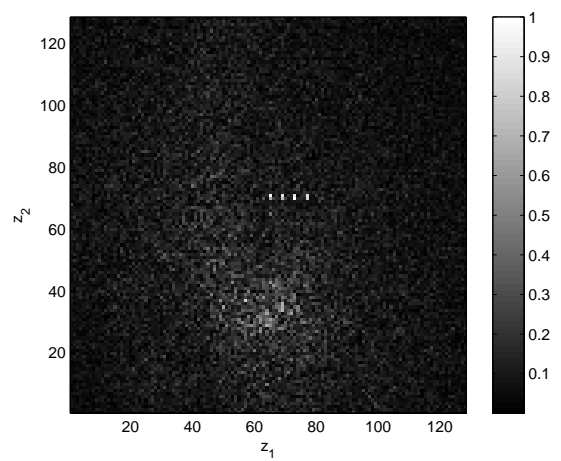

(c)

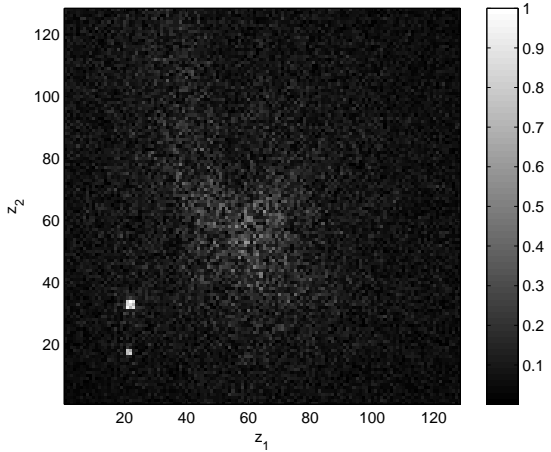

(b)

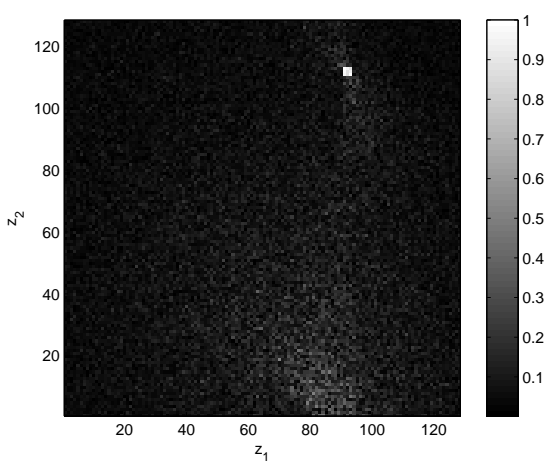

(d)

Figure 3: Reconstructed images with the target velocities: (a) $\mathbf{v}_{h}=[0,0,0] \mathrm{m} / \mathrm{s} ;$ (b) $\mathbf{v}_{h}=[-10,15,0] \mathrm{m} / \mathrm{s} ;$ (c) $\mathbf{v}_{h}=[0,10,0] \mathrm{m} / \mathrm{s} ;(\mathrm{d}) \mathbf{v}_{h}=[15,-5,0] \mathrm{m} / \mathrm{s}$.

\section{ACKNOWLEDGMENTS}

This work was supported by the Air Force Office of Scientific Research (AFOSR) under the agreements FA955007-1-0363 and FA9550-09-1-0013, and by the National Science Foundation (NSF) under Grant No. CCF08030672 .

\section{REFERENCES}

1. Werness, S., Carrara, W., Joyce, L., and Franczak, D., "Moving target imaging for SAR data," IEEE Transactions on Aerospace and Electronic Systems 26, 57-66 (Jan. 1990).

2. Friedlander, B. and Porat, B., "VSAR: A high resolution radar system for detection of moving targets," IEE Proceedings on Radar, Sonar and Navigation 144, 205-218 (1997).

3. Jakowatz, C. V., Wahl, J. D. E., and Eichel, P. H., "Refocus of constant velocity moving targets in synthetic aperture radar imagery," in [Proceedings of SPIE Conference on Algorithms for Synthetic Aperture Radar Imagery V], 3370, 85-95 (1998).

4. Perry, R. P., Dipietro, R. C., and Fante, R. L., "SAR imaging of moving targets," IEEE Transactions on Aerospace and Electronic Systems 35, 188-200 (Jan. 1999).

5. Fienup, J. R., "Detection moving targets in SAR imagery by focusing," IEEE Trans. on Aerospace and Electronic Systems 37, 794-801 (July 2001).

6. Jao, J. K., "Theory of synthetic aperture radar imaging of a moving target," IEEE Trans. on Geoscience and Remote Sensing 39, 1984-1992 (September 2001). 
7. Soumekh, M., "Moving target detection and imaging using an x band alon-track monopulse sar," IEEE Transactions on Aerospace adn Electronic Systems 38(1), 315-333 (2002).

8. Chen, V. C., "Radar detection of multiple moving taragets in clutter using time-frequency-radon transform," in [Proceedings of SPIE Conference on Signal and Data Processing of Small Targets], 4728, 48-59 (2002).

9. Kirscht, M., "Detection and imaging of arbitrarily moving targets with single-channel SAR," IEE Proc. on Radar Sonar Navig. 150, 7-11 (February 2003).

10. Pettersson, M. I., "Detection of moving targets in wideband SAR," IEEE Transactions on Aerospace and Electronic Systems 40, 780-795 (July 2004).

11. Minardi, M. J., Gorham, L. A., and Zelnio, E. G., "Ground moving target detection and tracking based on generalized SAR processing and change detection," in [Proc. of SPIE on Defense, Security and Sensing, Bellingham, WA, USA], 5808, 156-165 (April 2005).

12. Meta, A., Hoogeboom, P., and Ligthart, L. P., "Moving target indication enhancement in FMCW SAR using deramped randomized stepped-frequency signals," in [Proceedings of 2006 Waveform Diversity and Design Conference WDDC'06, Lihue, HI, USA], (Jan. 2006).

13. Cerutti-Maori, D., Klare, J., Brenner, A. R., and Ender, J. H. G., "Wide area traffic monitoring with the SAR/GMTI system PAMIR," IEEE Transctions on Geoscience and Remote Sensing 46, 3019-3030 (Oct. 2008).

14. Hack, D. E. and Saville, M. A., "Analysis of SAR moving grid processing for focusing and detection of ground moving targets," in [Proc. of SPIE on Defense, Security and Sensing, Orlando, FL, USA], 8051 (April 2011).

15. Deming, R. W., "Along-track interferometry for simultaneous SAR and GMTI: Application to gotcha challenge data," in [Proceedings of SPIE Conference on Algorithms for Synthetic Aperture Radar Imagery XVIII], 8051, P1-P18 (2011).

16. Vu, D., Guo, B., Zhou, L., and Li, J., "Ground moving target indication via multi-channel airborne SAR," in [Proceedings of SPIE Conference on Algorithms for Synthetic Aperture Radar Imagery XVIII], 8051, Q1-Q12 (2011).

17. Zhu, S., Liao, G., Qu, Y., Zhou, Z., and Liu, X., "Ground moving targets imaging algorithm for synthetic aperture radar," IEEE Trans. on Geoscience and Remote Sensing 49, 462-476 (January 2011).

18. Wang, L. and Yazıc1, B., "Bistatic synthetic aperture radar imaging of moving targets using ultranarrowband continuous waveforms," Inverse Problems (in preparation) (2012).

19. Wang, L. and Yazıc1, B., "Bistatic synthetic aperture radar imaging using ultra-narrowband continuous waveforms," IEEE Trans. on Image Processing (to appear, already available on IEEE website) . 